\title{
Salvage penile prosthetic surgery utilizing temporary malleable implants
}

\author{
Michael Lao ${ }^{1}$, R. James Graydon ${ }^{2}$, Jared M. Bieniek ${ }^{2}$ \\ ${ }^{1}$ Department of Urology, University of Connecticut Health Center, Farmington, CT, USA; ${ }^{2}$ Tallwood Urology \& Kidney Institute, Hartford \\ Hospital, Hartford, CT, USA \\ Contributions: (I) Conception and design: All authors; (II) Administrative support: None; (III) Provision of study materials or patients: All authors; \\ (IV) Collection and assembly of data: All authors; (V) Data analysis and interpretation: All authors; (VI) Manuscript writing: All authors; (VII) Final \\ approval of manuscript: All authors. \\ Correspondence to: Jared M. Bieniek. Tallwood Urology \& Kidney Institute, Hartford Hospital, 85 Seymour Street, Suite 416, Hartford, CT 06106, \\ USA. Email: jared.bieniek@hhchealth.org.
}

\begin{abstract}
The inflatable penile prosthesis (IPP) is an effective erectile dysfunction (ED) treatment modality when oral and injectable therapies fail to achieve satisfactory results. Unfortunately, infection of the prosthetic remains a dreaded complication occurring in a small fraction of patients despite advances in device design and surgical techniques. With a prosthetic infection or erosion, classic management has included removal of all hardware with thorough irrigation of the infected spaces. To prevent corporal fibrosis and scarring that can make a subsequent implant challenging, an immediate salvage procedure with a three-piece prosthesis has been advocated when possible. However, there has been recent interest in using malleable devices during salvage procedures to serve as a temporary implant and further improve outcomes. Based on a literature review of immediate salvage procedures for infected penile prostheses, management with typical Mulcahy washout and IPP reimplant may be quite successful in appropriately selected patients. Based on one case series and a second multicenter trial of malleable salvage procedures, utilizing a malleable as a temporary implant is similarly, if not more, successful at eradicating prosthetic infection. The malleable implant not only serves as a temporary space-filling corporal implant to prevent fibrosis, but may also prove an adequate destination therapy for some given the lower than expected rate of delayed conversion to inflatable prosthesis. Future studies are needed to better characterize the role of malleable devices for penile prosthetic salvage and query patient satisfaction with the malleable device and repeated surgeries.
\end{abstract}

Keywords: Erectile dysfunction (ED); infection; penile prosthesis; postoperative complication; salvage therapy

Submitted Feb 02, 2017. Accepted for publication Apr 25, 2017.

doi: $10.21037 /$ tau.2017.05.12

View this article at: http://dx.doi.org/10.21037/tau.2017.05.12

\section{Introduction}

Erectile dysfunction (ED) is a disorder of various etiologies related to vascular, neurogenic, psychologic, and pharmacologic factors with the most recalcitrant cases often occurring as the result of pelvic surgery such as radical prostatectomy (1). ED itself has been linked to negative self-perception, depression, marital problems, and suggestive of adverse cardiovascular health (2). When available treatment options including oral medications, intraurethral agents, intracavernosal injections, and vacuum devices prove ineffective or intolerable, the inflatable penile prosthesis (IPP) becomes the gold standard treatment choice for restoration of functional erections.

Early iterations of intracavernosal rods were made of various materials such as acrylic or polyethylene before the arrival of silicone rubber, creating a more 'natural' prosthesis (3). Dr. Brantley Scott revolutionized the treatment of ED in 1973 with the three-piece silicone IPP consisting of a reservoir, pump, and Dacron-reinforced 
corporal cylinders. Over the years, the IPP has undergone many improvements making it a popular choice amongst urologists with over 22,000 implants placed annually in the United States (4). Despite excellent efficacy and user satisfaction, however, IPP infections remain one of the most feared complications by patients and surgeons alike $(5,6)$.

IPP infection rates have decreased since device inception due to innovations such as coated implants and advances in surgical technique. In addition, multiple patient and operative factors related to infection rates have been identified (7). Patient influences such as smoking and diabetes can compromise wound healing and increase the overall rate of infection. Operative factors such as prophylactic antibiotics, chlorhexidine scrub, antibioticimpregnated implants, and the 'no-touch' technique have all been utilized in attempts to decrease infectious complications. Even with these precautionary measures, reported infection rates vary between $1-3 \%$ for newly implanted prostheses (8).

Infectious complications put a strain on the patient and physician, typically requiring repeat operative procedures. Antibiotic therapy alone is ineffective secondary to the formation of a bacterial or fungal biofilm around the foreign materials of the prosthetic which limits blood flow and acts as a protective cavity in which infectious agents thrive (9). Biofilms have proven to be a major problem in eradicating IPP infection, often making prosthetic explantation inevitable. In this review, we briefly look at the traditional management and salvage strategies for management of an infected IPP followed by a review of published data on malleable salvage procedures in an effort to determine today's most appropriate operative strategy.

\section{Traditional management of infected IPP}

Historically, typical management when IPP infection is suspected includes antibiotic treatment, removal of all prosthetic components, and a thorough wash out of the retropubic space (or other ectopic reservoir location), scrotum, and corpora cavernosa. Attempted reimplantation may then be considered several months later when the patient is rendered infection-free. Unfortunately, removal of the device will lead to fibrosis and scarring of the corporal bodies secondary to the inflammatory process involved. This fibrotic reaction results in penile shortening and makes subsequent insertion of a second IPP more challenging and prone to future infections and other complications. Patient satisfaction after reimplantation, while still high, is noticeably lower after such complications (10). The options to achieve erection other than another implant are virtually non-existent.

As a variant of traditional management, surgeons have tried inserting corporal irrigating drains to eradicate the infection and also prevent fibrosis $(11,12)$. The drains were irrigated with an antibiotic solution and the patient was encouraged to use a vacuum erection device to maintain penile length until later replacement of an IPP. Others have reported a sub-acute replacement strategy, or so-called "delayed salvage" procedure discussed further below, with new prosthesis implantation within $72 \mathrm{~h}$ of the explant in hopes of minimizing scarring (13).

\section{Mulcahy salvage protocol}

Mulcahy revolutionized the management of infected IPPs with his seminal work in 1996 assessing the feasibility of immediate replacement of an IPP at the time of salvage surgery (14). The initial retrospective paper described their experience with 11 patients with infected IPPs excluding device extrusions and poorly controlled diabetics with purulent infections. After removal of all hardware and foreign material, a seven-step antibiotic irrigation (bacitracin/kanamycin, half-strength hydrogen peroxide, half-strength povidone-iodine, pressure irrigation with vancomycin/gentamycin, followed by first three solutions in reverse order) was performed before a new prosthesis was inserted. Over a mean follow-up of 21 months, 10 of $11(91 \%)$ patients remained infection-free with a functional implant. As reported in other studies, the most common organisms cultured were skin flora such as gram positive Staphylococcus epidermidis $(8,15)$. Results from the original Mulcahy salvage series in addition to other salvage cohorts are summarized in Table 1.

Mulcahy further published his long-term experience with immediate salvage of 65 men with infected IPPs utilizing a similar washout protocol (16). Of 55 men with average follow-up of 35 months, 45 (82\%) had no further signs of infection. In the other 10 patients, 5 presented early with a repeat infection within one month of salvage surgery and the remaining 5 developed erosion of various components of their prosthetics. The authors concluded that immediate salvage can be a successful management approach to the infected IPP albeit with some failures.

Other groups have tried to replicate the long-term $82 \%$ success rate of the Mulcahy technique. Using the irrigation protocol and immediate reimplant, Kaufman 
Table 1 Success rates of immediate, short-term delayed, and malleable salvage of infected penile prostheses

\begin{tabular}{|c|c|c|c|c|c|c|}
\hline Study & Year & Technique & $\begin{array}{l}\text { No. of salvage } \\
\text { procedures }\end{array}$ & $\begin{array}{c}\text { No. of } \\
\text { infection-free [\%] }\end{array}$ & $\begin{array}{l}\text { Mean follow-up } \\
\text { (months) }\end{array}$ & $\begin{array}{c}\text { No. of converted } \\
\text { to IPP }\end{array}$ \\
\hline Brant et al. (14) & 1996 & Immediate salvage & 11 & 10 [91\%] & 21 & N/A \\
\hline Kaufman et al. (17) & 1998 & Immediate salvage & 7 & $6[86 \%]$ & 17 & N/A \\
\hline Köhler et al. (18) & 2009 & Malleable salvage & 6 & 6 [100\%] & 24 & $2(3 \text { considering })^{\dagger}$ \\
\hline Gross et al. (19) & 2016 & Malleable salvage & 58 & 54 [93\%] & 8.4 & 17 \\
\hline
\end{tabular}

${ }^{\dagger}$, one patient infection-free but had to get complete removal of malleable device due to near-erosion. N/A, not applicable; IPP, inflatable penile prosthesis.

et al. successfully treated 6 out of $7(86 \%)$ patients over a minimum follow-up of 13 months (17). Knoll et al. reported 8 of $10(80 \%)$ men who remained infection-free after immediate salvage with a mean follow-up duration of 11 months (13). In his series, Knoll compared an immediate salvage group $(\mathrm{n}=10)$ to a 3 -day delayed reimplantation cohort $(\mathrm{n}=31)$ for infected IPPs. Salvage was successful in 22 of $31(71 \%)$ delayed patients versus 8 of $10(80 \%)$ immediate replacement patients. Their analysis showed no significant differences between the two approaches. Due to potential savings in healthcare expenditure, the authors advocated for consideration of immediate salvage over a 3-day delay.

\section{Malleable rod salvage}

\section{Malleable salvage theory}

Despite the reported success with immediate salvage Mulcahy washout procedures, recurrent infections or complications may be observed in nearly $20 \%$ of men. Additionally, many of the reported studies excluded patients with purulent infections or device erosions. In an effort to offer salvage procedures for these men and further reduce prosthetic infections, some clinicians have begun using malleable rods as either a transition to eventual IPP replacement or, alternatively, as a final or destination therapy.

A benefit to malleable prosthetic salvage is the shorter operative time required in comparison to placement of a traditional three-piece prosthetic. Extra time in the operative suite may increase exposure to circulating pathogens and lead to device infection as suggested in other studies (20). Implantation of the three components of an inflatable prosthesis also requires additional manipulation of the scrotal or infrapubic incision, potentially exposing the device to more skin contact and thus transfer of normal flora that commonly lead to a prosthetic infections.

Malleable rods may offer the ideal middle ground to traditional infected IPP explant and a full washout and replacement of three-piece inflatable device. The rods maintain corporal patency after an infection and prevent scarring and penile shortening. Malleable devices also do not require a scrotal component and are ideally suited for men with scrotal pump erosions or purulence found at the time of exploration. Anecdotally, clinicians are beginning to utilize malleable rod salvage procedures for the reasons above with initial reports demonstrating good results.

\section{Reported results}

In a multicenter retrospective study conducted by Gross et al., study authors analyzed the malleable implant salvage technique (MIST) (19). The goal was to analyze outcomes after removal of an infected IPP and malleable replacement and assess the feasibility of delayed conversion back to an inflatable prosthesis. The study included 58 patients who underwent the Mulcahy irrigation protocol with removal of their IPP and replacement with an antibiotic-soaked malleable device (Coloplast Genesis or AMS Spectra) in the same operative setting between 2002 and 2014. The average salvage procedure took $148 \mathrm{~min}$ and occurred 2.8 months after the last prosthesis surgery. The mean post-operative follow-up was 8.4 months with the range extending out to 84 months. While a relatively short mean follow-up period, prior studies have shown that most infections typically 
occur within the first 12 months of implantation (21).

The multicenter cohort was composed of 42 men with infections after virgin implants and 16 with prior prosthetics (average of 2.6 prior prosthetic surgeries). At the time of their most recent follow-up, 54 of 58 (93\%) patients were infection-free. Of the 42 virgin implant infections, 38 (90\%) men underwent MIST without further infectious complications. Interestingly, despite prior surgeries, none of the 16 patients with prior prosthetics suffered any postoperative infectious complications after MIST. Once again, the most common organisms cultured during salvage surgery were skin flora. Forty-four men had available culture data with 15 (34\%) demonstrating coagulasenegative Staphylococcus. Unfortunately, four patients had persistent infection that required eventual removal of their malleable device occurring an average of 1 month after the salvage procedure. Of these 4 patients, 3 were initially virgin implants and one had prior penile surgeries.

The authors concluded that IPP infection can be safely and reliably treated with removal of infected hardware, washout, and insertion of a malleable prosthesis. This sequence was accompanied by a relatively low failure rate of $7 \%$ when compared to an $18 \%$ infection rate reported in Mulcahy's long-term IPP salvage series and other similar cohorts in the literature. Malleable prostheses may offer the benefit of preventing corporal scarring while reducing the complexity and number of components replaced at the time of salvage surgery.

Spinal cord injury (SCI) patients with neurogenic ED deserve special attention when discussing penile prostheses due to the concern for increased complications, especially with malleable devices. Many SCI men will choose a penile prosthetic for treatment of their ED with some preferring a malleable device due to limited hand dexterity. Malleable devices continually apply some degree of pressure at the proximal and distal tips of the corporal rods. If SCI men cannot feel the device due to an insensate penis, they may not recognize early symptoms of rod extrusion until the prosthetic is visualized outside the penis. Another potential concern is the risk of urethral injury or bacterial translocation with indwelling or intermittent catheterization in SCI men with associated neurogenic bladders. These concerns however are tempered by low complication rates in studies including a long-term review of 48 SCI men with malleable prostheses over 11.7 years of follow-up (22). Overall complications occurred in $8(16.7 \%)$ patients including $4(8.3 \%)$ with device infections and $2(4.2 \%)$ with erosions. This low rate of malleable device extrusion in SCI men with over one decade of follow-up is reassuring and should not exclude the use of malleable salvage techniques in such men.

Malleable salvage may also be an ideal strategy for men with isolated scrotal pump infectious complications. Revision of the scrotal pump alone will not remove infectious agents that may have colonized the tubing and other device components. Additionally, the already compromised scrotal skin may be more prone to device extrusion if a pump is reimplanted. In an effort to reduce repeat complications in this population, Köhler et al. reviewed their experience with scrotal pump erosions or infections managed with malleable substitutions (18). The study retrospectively reviewed six men who underwent removal of IPP for an infected or eroded scrotal pump with malleable rod implantation. All patients remained infection-free after malleable implant with a mean follow-up of 2 years. One patient developed impending erosion of his malleable rod and underwent elective device removal. The authors concluded that malleable substitution is an excellent option for the management of scrotal pump erosion or infection.

In our experience, another candidate for possible malleable salvage is the man with a history of complicated penile prosthesis procedures who presents with no device in situ. At that point, his corpora are likely fibrosed, making reimplantation of a three-piece device challenging and requiring extensive dissection, various corporal and skin incisions, and, perhaps most importantly, prolonged operative time and infectious risk. In such cases, we often preoperatively discuss the potential for a temporary malleable implant if the procedure duration exceeds an acceptable length of time. We then offer delayed conversion to an IPP a few months later if the patient wishes. We are continuing to collect data on a group of similar men in our practice while following their outcomes.

Further extrapolating upon the space-filling theory of a malleable salvage, Swords et al. reported their initial experience with an intracorporal antibiotic cast for treatment of infected penile implants in two men (23). Often used in orthopedics as a bone space filler, calcium sulfate $\mathrm{CaSO}_{4}\left(\right.$ Stimulan $\left.^{\circledR}\right)$ is prepared as a paste which hardens over time. As a corporal space filler for penile infection, it can be mixed with antibiotics and injected into the corporal spaces, dissolving over a period of approximately 4-6 weeks. In their series, Swords et al. mixed the calcium sulfate with vancomycin and tobramycin to form antibiotic-laden corporal casts. Delayed reimplantation of a new prosthetic should ideally be performed before full resorption of the 
cast to prevent penile fibrosis from occurring.

In Swords' case series, one man presented with erosion of his right malleable implant with purulent drainage. The eroded rod was removed and the corporal space washed out with antibiotic solution before injecting the calcium sulfate/antibiotic paste and molding the penis. By 2 weeks postoperatively, the cast had hardened and felt like a malleable device. The cast began resorbing by 4 weeks and reimplantation was performed at 6 weeks with no evidence of penile fibrosis. At 6 months, he was able to have satisfactory intercourse with no signs of infection. The second patient had a history of multiple penile surgeries including placement of an IPP with later explantation for infection and placement of malleable rods. He further underwent revision surgery for continued pain where significant scar was encountered during removal of his rods. The corpora were irrigated and the calcium sulfate mixture utilized to create bilateral corporal casts. He was lost to follow-up with delayed reimplantation eventually performed at 15 weeks. By that time, the cast had completely resorbed and significant fibrosis was present. Only a unilateral malleable rod was able to be safely placed although the patient reported satisfactory intercourse at 2-month follow-up. Significant corporal scarring noted at 15 weeks suggests that delayed reimplantation may be better accomplished before full dissolution of the cast. The authors concluded that this option may be best suited for the high-risk patient that is acutely sick or septic, has cylinder extrusions, history of recurrent infections, or is not a candidate for an immediate salvage procedure. Importantly, the cast is strictly temporary and cannot be used for sexual activity. This preliminary report is quite thought provoking and suggests that more cost-effective temporary implants (rather than malleable rod prostheses) may be an option for men who plan to undergo a delayed inflatable prosthetic replacement.

Despite the excellent results demonstrated with Mulcahy salvage and especially with malleable rod salvage, a recent study by Zargaroff $e t$ al. analyzing the national trends in treatment of IPP infections found a surprisingly low rate of salvage procedures are being performed (24). Utilizing the Nationwide Inpatient Sample database from 2000 to 2009, they reported that $82.7 \%$ of infected IPPs were treated with explantation alone while only $17.3 \%$ underwent an immediate salvage procedure. Patients undergoing immediate salvage were typically younger and had a less severe presentation. The age difference may reflect patient wishes or surgeon bias towards selecting younger, healthier patients for reoperative procedures. Hospital location also had a bearing on treatment choice with lower odds of immediate salvage at rural hospitals compared to urban teaching hospitals. Although salvage techniques are still considered by many high-volume implanters to be the gold standard for treatment of infected IPPs, this study suggests that salvage procedure are underutilized.

\section{Delayed conversion to IPP}

When discussing a malleable salvage procedure with a patient with an infected IPP, the malleable prosthetic is usually described as a bridge to eventual replacement with the more natural-appearing three-piece prosthesis. Surprisingly, however, 37 of the 54 (69\%) infection-free patients in the Gross et al. series still had their malleable device in place at their most recent follow-up (19). Seventeen $(31 \%)$ men elected to undergo replacement of their malleable device with an inflatable prosthesis occurring on average 6.7 months after their initial washout surgery. The authors found that the patients who elected IPP replacement were typically younger by 10 years which is in line with the findings by Zargaroff et al. (24). The average follow-up for Gross' multicenter cohort was only 8.4 months so longer follow-up may find a higher proportion of men proceeding with IPP replacement. Alternatively, men may be satisfied with their malleable device for functional intercourse and not desire repeat surgery. This final point speaks to the limitations of this approach by requiring subsequent surgery to replace the malleable which puts the patient at risk of repeat anesthetic, infectious, and other device-related complications.

In the Köhler series of scrotal pump erosions, 2 of the 6 patients elected to convert back to IPP after a malleable salvage (18). Another three patients were planning future conversion back to IPP at the time of publication. The lone remaining patient had near erosion of a malleable rod and underwent complete device removal. The authors similarly suggest that there appears to be some satisfaction with the functionality of the malleable prostheses among the men in their series though most have or are planning to eventually convert to an IPP.

It would be interesting to survey patients' perceived and actual experiences with malleable and inflatable prostheses during various stages of malleable implant salvage. This information could shed light on whether men are indeed satisfied with their malleable as a final treatment option, fearful of repeat surgery and resigned to living with a 
malleable, or planning later replacement with an IPP. Long-term outcomes of prospectively collected data will additionally help to define the role of malleable salvage in the world of penile prosthetics.

\section{Conclusions}

Treatment of infected IPPs has gone through various revisions and advances. While traditional management necessitates complete device removal with possible attempts at delayed replacement, updated series have shown good results with Mulcahy salvage and immediate inflatable prosthesis replacement. Anecdotally, high-volume prosthetic surgeons have started to move towards a middleground approach, utilizing a malleable salvage technique to serve as a temporary space-filling corporal implant. Recent series have demonstrated very good infection-free rates with malleable salvages, perhaps slightly better than standard Mulcahy salvage. Interestingly, some men appear satisfied with their malleable device and choose not to go on to eventual IPP replacement. Further data is needed on the safety and outcomes with a malleable salvage approach to infected IPPs. Specific attention should be paid to patient opinions and experiences so that we can appropriately counsel men during this challenging process.

\section{Acknowledgements}

None.

\section{Footnote}

Conflicts of Interest: The authors have no conflicts of interest to declare.

\section{References}

1. Henry GD, Karpman E, Brant W, et al. The Who, How and What of Real-World Penile Implantation in 2015: The PROPPER Registry Baseline Data. J Urol 2016;195:427-33.

2. Miner M, Nehra A, Jackson G, et al. All men with vasculogenic erectile dysfunction require a cardiovascular workup. Am J Med 2014;127:174-82.

3. Le B, Burnett AL. Evolution of penile prosthetic devices. Korean J Urol 2015;56:179-86.

4. Montague DK. Penile prosthesis implantation in the era of medical treatment for erectile dysfunction. Urol Clin North Am 2011;38:217-25.
5. Carson CC, Mulcahy JJ, Govier FE. Efficacy, safety and patient satisfaction outcomes of the AMS 700CX inflatable penile prosthesis: results of a long-term multicenter study. AMS 700CX Study Group. J Urol 2000;164:376-80.

6. Mulhall JP, Ahmed A, Branch J, et al. Serial assessment of efficacy and satisfaction profiles following penile prosthesis surgery. J Urol 2003;169:1429-33.

7. Holland B, Kohler T. Minimizing Penile Implant Infection: A Literature Review of Patient and Surgical Factors. Curr Urol Rep 2015;16:81.

8. Carson CC. Infections in genitourinary prostheses. Urol Clin North Am 1989;16:139-47.

9. Nickel JC, Heaton J, Morales A, et al. Bacterial biofilm in persistent penile prosthesis-associated infection. J Urol 1986;135:586-8.

10. Caire AA, Boonjindasup A, Hellstrom WJ. Does a replacement or revision of an inflatable penile prosthesis lead to decreased patient satisfaction? Int J Impot Res 2011;23:39-42.

11. Maatman TJ, Montague DK. Intracorporeal drainage after removal of infected penile prostheses. Urology 1984;23:184-5.

12. Kim JC, Lunati FP, Khan SA, et al. T-tube drainage of infected penile corporeal chambers. Urology 1995;45:514-5.

13. Knoll LD. Penile prosthetic infection: management by delayed and immediate salvage techniques. Urology 1998;52:287-90.

14. Brant MD, Ludlow JK, Mulcahy JJ. The prosthesis salvage operation: immediate replacement of the infected penile prosthesis. J Urol 1996;155:155-7.

15. Blum MD. Infections of genitourinary prostheses. Infect Dis Clin North Am 1989;3:259-74.

16. Mulcahy JJ. Long-term experience with salvage of infected penile implants. J Urol 2000;163:481-2.

17. Kaufman JM, Kaufman JL, Borges FD. Immediate salvage procedure for infected penile prosthesis. J Urol 1998;159:816-8.

18. Köhler TS, Modder JK, Dupree JM, et al. Malleable implant substitution for the management of penile prosthesis pump erosion: a pilot study. J Sex Med 2009;6:1474-8.

19. Gross MS, Phillips EA, Balen A, et al. The Malleable Implant Salvage Technique: Infection Outcomes after Mulcahy Salvage Procedure and Replacement of Infected Inflatable Penile Prosthesis with Malleable Prosthesis. J Urol 2016;195:694-7.

20. Pineda M, Burnett AL. Penile Prosthesis Infections-A Review of Risk Factors, Prevention, and Treatment. Sex 
Med Rev 2016;4:389-98.

21. Carson CC. Diagnosis, treatment and prevention of penile prosthesis infection. Int J Impot Res 2003;15 Suppl 5:S139-46.

22. Kim YD, Yang SO, Lee JK, et al. Usefulness of a malleable penile prosthesis in patients with a spinal cord injury. Int J Urol 2008;15:919-23.

23. Swords K, Martinez DR, Lockhart JL, et al. A preliminary

Cite this article as: Lao M, Graydon RJ, Bieniek JM. Salvage penile prosthetic surgery utilizing temporary malleable implants. Transl Androl Urol 2017;6(Suppl 5):S806-S812. doi: 10.21037/tau.2017.05.12 report on the usage of an intracorporal antibiotic cast with synthetic high purity CaSO4 for the treatment of infected penile implant. J Sex Med 2013;10:1162-9.

24. Zargaroff S, Sharma V, Berhanu D, et al. National trends in the treatment of penile prosthesis infections by explantation alone vs. immediate salvage and reimplantation. J Sex Med 2014;11:1078-85. 